TAO, Vol. 14, No. 2, 221-240, June 2003

\title{
A Brief Review of Space Weather Disturbances
}

\author{
Anthony T. Y. Lui ${ }^{1, \star}$ \\ (Manuscript received 1 May 2003, in final form 2 June 2003)
}

\begin{abstract}
A branch of physics which becomes increasingly relevant to our societal functions is magnetospheric physics. This rise to fame is due to the incessant growth in the utilization of space technology and assets in our daily lives. Much like adverse atmospheric weather can wreak havoc to our homes and facilities, disturbances in the magnetosphere can render space assets for communication and navigation inoperative as well as pose hazards to astronauts and people on commercial polar flights. Two main space disturbances are magnetic storms and magnetospheric substorms. The former has been studied for more than a century and the latter for four decades. This article provides a brief overview of the main features observed in these space disturbances and some theoretical models for their cause.
\end{abstract}

(Key words: Space weather, Magnetic storms, Magnetospheric substorms)

\section{INTRODUCTION}

Mankind is generally mesmerized by colorful natural phenomena. We marvel and often pause whatever we are doing at the sight of a rainbow. We ponder about how it is formed. We invent fascinating folklore and compose songs about it. These human traits are indications of our desire to understand nature and our effort to comprehend our place in the universe.

Another case in point is the auroral display, which is less known by residents in the tropics but well recognized by those fortunately or unfortunately residing in the northern and southern polar regions. Auroras are mostly seen in the night sky, appearing in colors of blue, green, and red - the three primary colors used in the transmission of color television today. Emissions from molecular nitrogen give blue and red colors while those from atomic oxygen give green and red colors. The brightness can be comparable to that of the full moonlight. They come in various forms, as very quiescent curtain-like shapes stretching from horizon to horizon, or as rays emanating apparently from an invisible point, or as spirals occupying the entire sky, or as dynamic flickering and randomly distributed patches mimicking uncoordinated fireworks. One

\footnotetext{
${ }^{1}$ The Johns Hopkins University Applied Physics Laboratory, Laurel, MD 20723, USA

${ }^{\star}$ Corresponding author address: Prof. A. T. Y. Lui, The Johns Hopkins University Applied Physics Laboratory, Laurel, MD 20723, USA; E-mail: Tony.Lui@jhuapl.edu
} 
can also see auroras from space, as exemplified by photographs of auroras observed from a space shuttle. In the space physics discipline, we recognize that auroral display is a message in the sky, broadcasting the disturbance level of the invisible outer space in our neighborhood.

What is the origin of these disturbances in the polar regions? The answer would take us to well beyond the immediate Earth's environment - our Sun. Our Sun emits more than the electromagnetic radiation. Its atmosphere expands continuously outward, producing fast outflow of particles. Since the Sun's temperature is high enough to cause some of the electrons in atoms and molecules to escape freely from their nuclei, the outflow stream consists almost entirely of electrically charged particles (ions and electrons) commonly known as a plasma. This fast outflow plasma is called the solar wind. Occasionally, instead of a continuous outflow, our Sun will spurt out giant clouds of the hot plasma called coronal mass ejections. The complex interaction of the solar plasma (in the form of the wind or the coronal mass ejection) with the Earth's magnetic field is the ultimate energy source for the dynamic patterns of auroras in the polar sky.

Modern space exploration began with the pioneering launch of the artificial satellite Sputnik by Russia in 1957. Since then, thousands of man-made satellites have been launched into space to make both in situ and remote sensing observations. Technological assets in space are accumulating at an accelerating pace. With this growth comes our dependence on these space technologies for our daily routine functions, such as in communication, navigation, and global surveillance of atmospheric weather. Space science has ascended from mere academic interest to practical necessity as adverse space disturbances start to impact our daily activities. This sudden rise to fame is similar to that of atmospheric science at airplane altitudes when commercial air travel becomes accessible to our society.

This article presents a brief glimpse of two major space weather disturbances, namely, magnetospheric substorms and magnetic storms. Main emphasis here will be the observed characteristics of these two phenomena, followed by rather abridged overviews of some prominent theories proposed for their causes. This approach is chosen to be in line with the present state-of-the-art knowledge on these two topics since the physical processes responsible for their occurrence are still openly debated in the research literature.

This review is admittedly not all-inclusive in the sense that other space weather phenomena outside these two major space weather disturbances are not covered in here. Nevertheless, the content hopefully will be adequate for the general community to gain appreciation of some space-related disturbances and challenges faced by space scientists today.

\section{THE EARTH'S MAGNETOSPHERE}

A general description of the near-Earth space environment is in order before proceeding to discuss space disturbances. The basic features of the near-Earth space are illustrated in Fig. 1. As discussed in Section 1, the continuous expansion of the solar atmosphere generates the solar wind that engulfs our solar system. The magnetic field embedded in the solar wind is called the interplanetary magnetic field. Due to the high supersonic speed of the solar wind and the shield from the Earth's magnetic field, a shock wave called the bow shock is generated 
to deflect the oncoming solar wind from reaching the Earth's surface. Behind the bow shock, a magnetic bubble called the magnetosphere is formed. The surface of the magnetosphere is known as the magnetopause.

In the nightside, the Earth's magnetic field is stretched downstream to a long distance, forming the magnetotail much like the tail of a comet. The equatorial region of the magnetotail contains a sheet of plasma, known as the plasma sheet. The regions above and below the plasma sheet are known as the magnetotail lobes, which have a much lower particle density than that of the plasma sheet.

A number of electric current systems flow within the magnetosphere. In particular, the current system in the plasma sheet stretches from the dawn flank to the dusk flank of the magnetotail and is referred to as the cross-tail current. There is a continuous extension of this current system toward the Earth. This earthward extension is called the ring current since it appears in the inner magnetosphere as a donut-shaped region encircling the Earth.

\section{ELECTRIC CURRENTS IN SPACE}

Electric currents in space are key elements in magnetospheric substorms and magnetic storms. The ring current and the cross-tail current flow perpendicular to the magnetic field. They are driven by the gradient of plasma pressure, pressure anisotropy in curved magnetic field geometry, and the inertial force. Therefore, the current density perpendicular to the magnetic field in the magnetosphere $j_{\perp}$ can be expressed as

$$
\bar{j}_{\perp}=\frac{\vec{B}}{B^{2}} \times\left[\nabla P_{\perp}+\left(P_{\|}-P_{\perp}\right) \frac{\vec{B} \cdot \nabla \vec{B}}{B^{2}}+\rho \frac{d \vec{v}}{d t}\right],
$$

where $\bar{B}$ is the magnetic field, $P_{\perp}$ and $P_{\|}$are the plasma pressure components perpendicular and parallel to the magnetic field, $\rho$ is the plasma density, and $\vec{v}$ is the plasma velocity. Deep in the magnetosphere where the electric currents are the main element of magnetic storms, the inertia force is typically negligible and the pressure gradient term in Eq. (1) is generally larger than the term associated with the pressure anisotropy. Therefore, pressure gradient is the dominant force governing the perpendicular current density.

Electric currents also flow along magnetic field lines and they play a pivotal role in coupling the magnetosphere and the ionosphere. Typically, these parallel currents come from the divergence of perpendicular electric currents due to the constraint of quasi-charge-neutrality in a plasma, i.e., the number of positive charged particles equals approximately to the number of negative charged particles (typically electrons) in any given volume to avoid charge buildup leading to large electric fields. The divergence of perpendicular electric field in the magnetosphere also plays a role in the generation of parallel electric currents. Bright auroral displays are accompanied by parallel electric currents carried mostly by precipitating electrons from the magnetosphere. Therefore, it is essential to understand perpendicular currents and the associated electric field in the magnetosphere to comprehend the cause of bright auroras and 


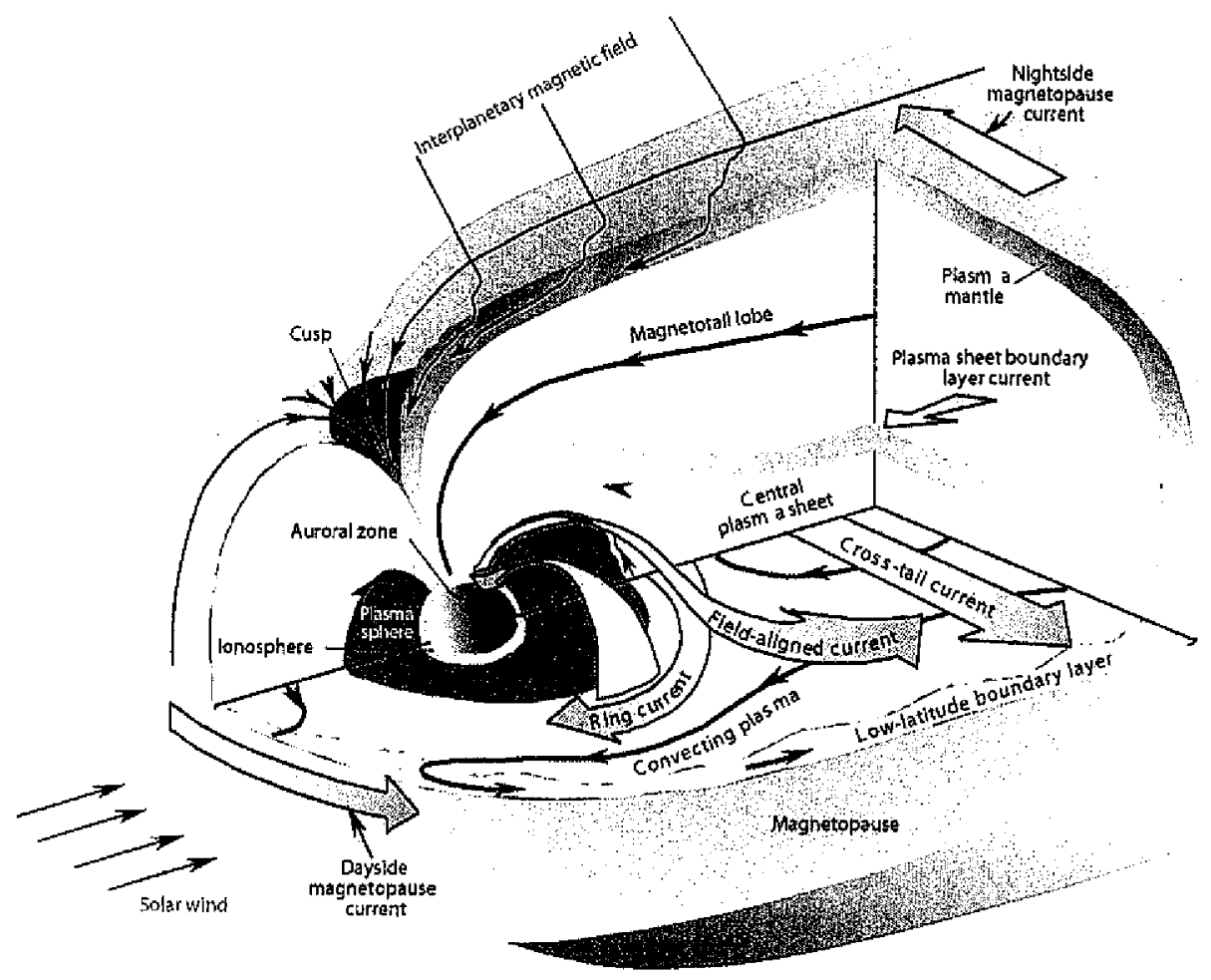

Fig. 1. A schematic diagram illustrating various features of the Earth's magnetosphere.

their associated parallel electric currents.

The famous Nobel laureate Hannes Alfvén introduced a powerful concept, the guiding center approximation, to visualize the motion of charged particles in space and leads to an elegant means of specifying how perpendicular currents can exist in space based on individual particle motion (Alfvén 1950). For a charged particle of mass $m$, charge $q$ and velocity $\vec{v}$, its equation of motion in the presence of a magnetic field $\vec{B}$ is

$$
m \frac{d \vec{v}}{d t}=q \vec{v} \times \vec{B} .
$$

The perpendicular component of Eq. (2) indicates that the perpendicular motion of the particle is circular with a gyroradius $m v_{\perp} / q B$ ( $v_{\perp}$ is the particle's velocity perpendicular to the magnetic field), a gyration period of $2 \pi \mathrm{m} / q B$, and the sense of gyration dependent on the sign of the charge. This gyration of the particle around the magnetic field due to the Lorentz force is analogous to the orbital motion of a planet around the Sun due to the gravitational force. The guiding center concept provides tremendous insight for particle motion in the presence of electric and magnetic fields even when the fields are inhomogeneous and time varying, pro- 
vided that the length scale of field inhomogeneity is larger than the particle's gyroradius and the time scale of field variations is longer than its gyration period. In the presence of an electric field $\vec{E}$, the particle has a convection motion given by

$$
\vec{v}_{E}=\vec{E} \times \vec{B} / B^{2}
$$

which is independent of charge, mass, and energy of the particle. In inhomogeneous magnetic field, this guiding center concept leads to quantitative expressions of particle drifts and their dependence on particle mass, energy, and charge. For example, a magnetic field gradient gives rise to a gradient drift motion of the particle

$$
\vec{v}_{g}=m v_{\lrcorner}^{2} \vec{B} \times \nabla B /\left(2 q B^{3}\right),
$$

which increases with mass and energy. Its drift direction depends on the sign of the charge. When the magnetic field has a curvature, it undergoes a curvature drift with similar characteristics as that of the gradient drift in terms of mass, energy, and charge, namely,

$$
\vec{v}_{c}=m v_{\|}^{2} \vec{B} \times(\vec{B} \bullet \nabla)(\vec{B} / B) /\left(q B^{3}\right),
$$

where $v_{\|}$is the particle's velocity parallel to the magnetic field. Both gradient and curvature drifts are charge dependent and thus constitute electric currents. An additional contribution of local current density comes from the gradient of the mass density and/or temperature of the particle population. This current arises from a denser concentration of gyration orbits (due to a mass density gradient) and/or larger gyration speeds (due to a temperature gradient) although there is no actual particle drift. The current, called the magnetization current, is given by

$$
\vec{j}_{m}=-\nabla \times \frac{\vec{B}}{B^{2}} \int \frac{m v_{\perp}^{2}}{2} f(\vec{v}) d^{3} v
$$

where $f(\vec{v})$ is the velocity distribution of the particle population in the velocity space.

\section{MAGNETOSPHERIC SUBSTORMS}

Substorm disturbances were recognized originally from studies of ground-based magnetograms in the polar regions. The horizontal component of magnetic field at high-latitudes shows large perturbations during substorms, a result of strong currents (of the order of one million amperes) flowing in the ionosphere. The strong ionospheric currents can induce large currents and voltages on the ground and in power transmission lines. They can also trip circuit breakers and damage transformers. This effect can lead to failure of power grids. A case in point is the power outage on 13 March 1989 in the Province of Quebec, Canada that 
lasted for nine hours.

A measure of substorm intensity, called the $\mathrm{AE}$ (auroral electrojet) index, was introduced by superposing perturbations of the horizontal component of the magnetic field measured at (twelve) longitudinally distributed magnetic stations in the polar region. The process responsible for these disturbances were initially perceived as an elementary building block of magnetic storms, hence the term "sub-storm". The identification of substorm occurrence was later defined more specifically based on studies of auroral pattern in the polar region from a network of all-sky cameras (Akasofu 1964). The study provides the view on the progression of aurora activity over the globe if one were able to watch it on a platform hovering above the polar region.

The evolution of auroras during substorms is illustrated in Fig. 2. Typically seen in the night sky before substorm onset are quiet auroral arcs oriented more or less parallel to magnetic latitudes. At substorm onset, one of the nightside auroral arcs brightens suddenly and breaks up into irregular patches. The disturbance expands poleward as well as to other local times. The expansion of aurora activity may last for about $1 \mathrm{hr}$ before it begins to subside. Substorms may occur so frequently that they overlap in time, such as during magnetic storms. In such a case, the temporal development of auroral pattem becomes more complex.

In the magnetosphere, energetic particle fluxes can increase abruptly from the transport of plasma sheet particles toward the Earth. These particles can interfere with satellite operations and may lead to failure of an entire satellite. Case in point is the loss of the AT\&T Telstar 401 satellite on 10 January 1997. Simultaneous with enhanced levels of energetic particle fluxes, the magnetic field exhibits large fluctuations indicative of turbulence onset. An example of measurements showing these changes is given in Fig. 3. The observed magnetic field changes in the magnetosphere, together with the horizontal magnetic perturbations on the ground at high latitudes, are consistent with the notion of short-circuiting a portion of the cross-tail current along magnetic field lines down to the low altitude polar ionosphere during substorms. This phenomenon of magnetic turbulence illustrated in Fig. 3 is called current disruption. In a sense, substorms could be viewed as an electrical discharge process much like lightning. Figure 4 is a schematic diagram to illustrate the analogy between them (Lui 1991). Both are natural phenomena associated with discharges of electricity. Each energy release in both phenomena is quite transient and spatially localized. However, the affected areas can be quite extensive in spite of the spatial confinement of each energy release event.

Two scenarios are being considered for the mechanism responsible for substorms, shown here in Fig. 5. The first scenario, the current disruption model, invokes some physical process acting close to the Earth. Several potential mechanisms are proposed in the literature under this scenario and are briefly discussed in the following.

One idea of current disruption is a plasma instability, known as the cross-field current instability (Lui et al. 1991), which limits the strength of the cross-tail current and shunts it to the ionosphere when the current density exceeds a certain threshold. In a simplified description, the process invokes waves, out of the thermal noise spectrum, being excited by the free energy from the strong current density. The current density reduces its strength as its energy is tapped by the excited waves. The current continuity condition as described in the previous section promotes the generation of currents along the magnetic field, thus setting up a current system 

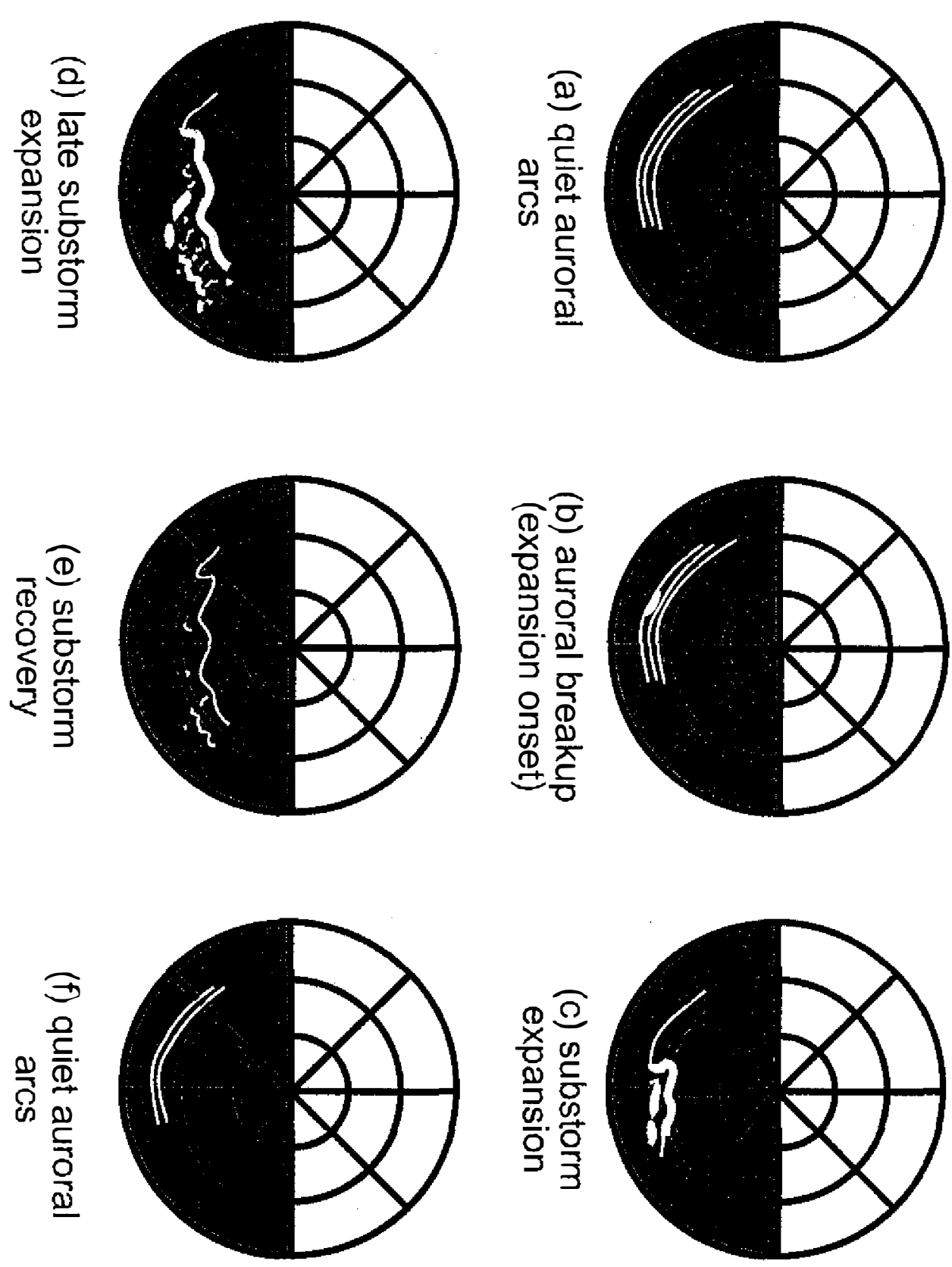

Fig. 2. An illustration of the progression of auroral activity during a magnetospheric substorm. 


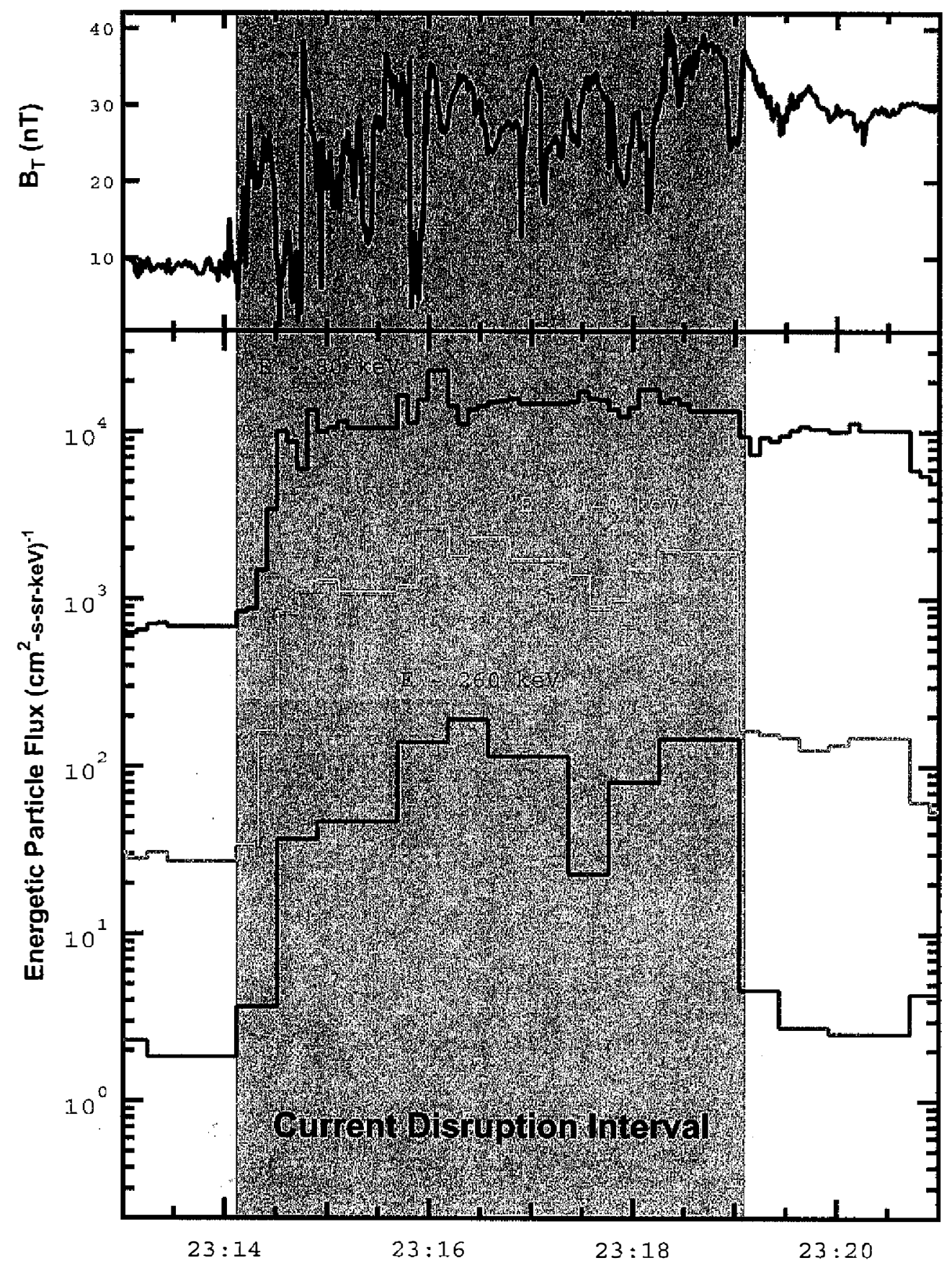

Fig. 3. A example of current disruption in the magnetosphere during which magnetic field fluctuates wildly and energetic particles increase tremendously in intensity. 


\section{ANALOGY BETWEEN TWO NATURAL PHENOMENA}

Lightning storm

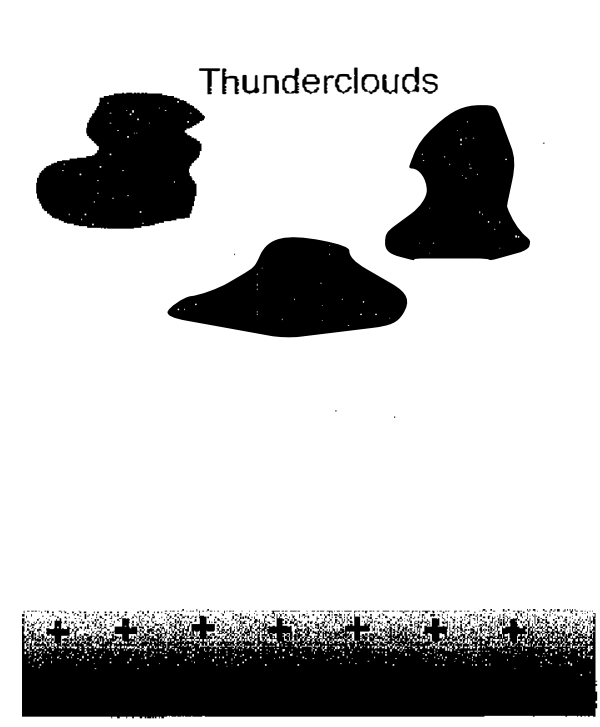

Magnetospheric Substorm

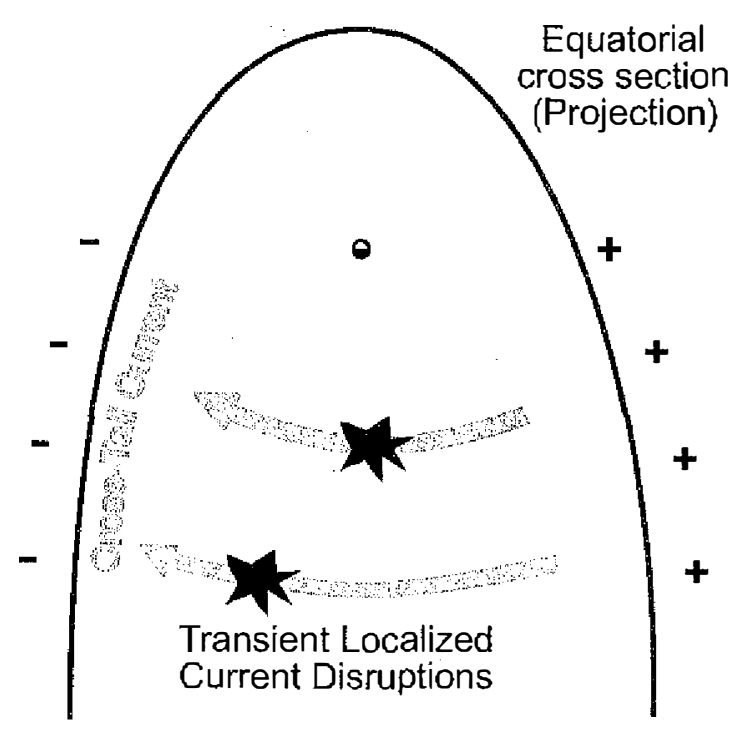

Fig. 4. Analogy between lightning storm and magnetospheric substorm. These two natural phenomena involve impulsive and spatially localized electrical discharges. For the magnetospheric substorm sketch, the portion of diversion of the cross-tail current into the ionosphere is indicated by yellow arrows. The dashed arrows indicate a subsequent current diversion instigated by an adjacent earlier current disruption.

tantamount to short-circuiting the cross-tail current. Particles are transported earthward as the magnetic field in the current disruption region relaxes from the reduction of the local current density. Another consequence of the plasma instability is the acceleration of electrons along the magnetic field, allowing them to precipitate into the polar ionosphere and cause the visual auroras. The initial disturbance of current disruption propagates as a rarefaction wave down the magnetotail (Chao et al. 1997) and instigates other regions downstream in the magnetotail to be activated, spreading the activity like an avalanche and giving rise to a large-scale disturbance for substorms.

In general, the evaluation on the role of plasma instability in a given system utilizes the Newton's equation of motion and the Maxwell's equations to derive a dispersion relation. The perturbation quantities are assigned a spatiotemporal dependence of $e^{i(\vec{k} \bullet \vec{r}-\omega t)}$, where $\vec{k}$ is the wavenumber, $\vec{r}$ is the distance vector, $\omega$ is the wave frequency, and $t$ is the time. The dispersion relation is usually in the form of $\ddot{D}(\vec{k}, \omega) \bullet \vec{U}=0$, where $\vec{D}(\vec{k}, \omega)$ is a square matrix dependent on $\vec{k}$ and $\omega$ while $\vec{U}$ is a vector representing the perturbed quantity such as the 


\section{Two Substorm Onset Models}

\section{Current Disruption Model}

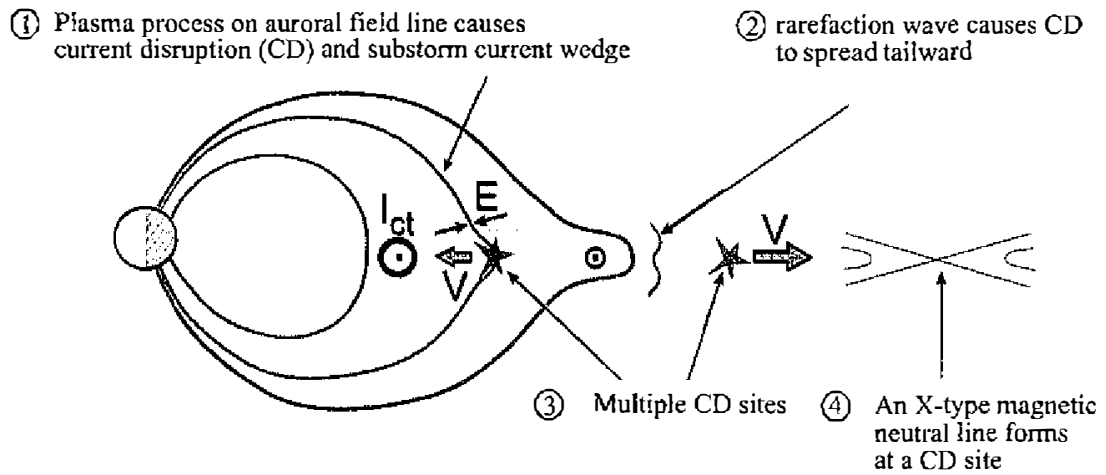

Near-Earth Neutral Line Model

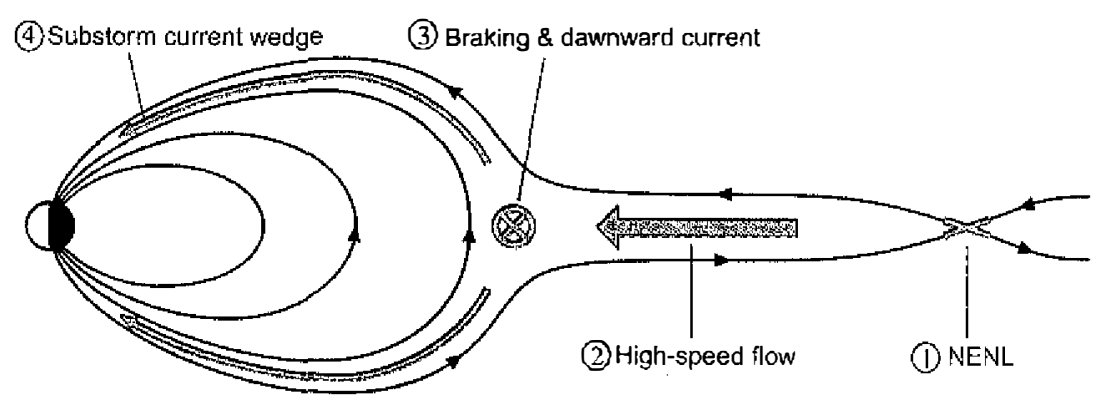

Fig. 5. A diagram to describe two substorm onset models under consideration in the literature.

electric field, magnetic field, vector displacement, or vector potential. The dispersion equation is given by $|\vec{D}(\vec{k}, \omega)|=0$. If the solution of the dispersion equation gives a positive value for the imaginary part of $\omega$, then the wave will grow in time from the thermal noise. For example, considering wave propagation in the $y z$-plane (the typical coordinate system used in space is that $x$-axis points to the Sun, $y$-axis to the dusk, and $z$-axis to the north; this coordinate system will be adopted hereafter) and ions drifting in the $y$-direction, with $\vec{U}$ being the electric field perturbation, one can show that the components of the determinant for the cross-field current instability in the dispersion equation are (Lui et al. 1991)

$$
D_{x x}=1-k^{2} c^{2} / \omega^{2}+\frac{\omega_{p i}^{2}}{\omega^{2}} \xi_{i} Z\left(\xi_{j}\right)+\frac{\omega_{p e}^{2}}{\omega^{2}} e^{-\mu} \xi_{e} \sum_{n=-\infty}^{\infty}\left\{\frac{n^{2}}{\mu} I_{n}(\mu)-2 \mu\left[I_{n}^{\prime}(\mu)-I_{n}(\mu)\right]\right\} Z\left(\xi_{n}\right)
$$




$$
\begin{aligned}
D_{y y}= & 1-k_{z}^{2} c^{2} / \omega^{2}+\frac{\omega_{p i}^{2}}{\omega^{2}}\left\{\xi_{i} Z\left(\xi_{i}\right) \cos ^{2} \theta-\left[\xi_{i} \sin \theta+v_{o} / v_{i}\right]^{2} Z\left(\xi_{i}\right)\right\} \\
& +\frac{\omega_{p e}^{2}}{\omega^{2}} \frac{e^{-\mu}}{\mu} \xi_{e} \sum_{n=-\infty}^{\infty} n^{2} I_{n}(\mu) Z\left(\xi_{n}\right), \\
D_{z z}= & 1-k_{y}^{2} c^{2} / \omega^{2}+\frac{\omega_{p i}^{2}}{\omega^{2}} \xi_{i}\left\{Z\left(\xi_{i}\right) \sin ^{2} \theta-\xi_{i} Z^{\prime}\left(\xi_{i}\right) \cos ^{2} \theta\right\} \\
& \div \frac{\omega_{p e}^{2}}{\omega^{2}} 2 \xi_{e}\left[\xi_{e}+e^{-\mu} \sum_{n=-\infty}^{\infty} \xi_{n}^{2} I_{n}(\mu) Z\left(\xi_{n}\right)\right] \\
D_{x y}= & -D_{y x}=i \frac{\omega_{p e}^{2}}{\omega^{2}} e^{-\mu} \xi_{e} \sum_{n=-\infty}^{\infty}\left\{n\left[I_{n}^{\prime}(\mu)-I_{n}(\mu)\right]\right\} Z\left(\xi_{n}\right), \\
D_{x z}= & -D_{z x}=i \frac{\omega_{p e}^{2}}{\omega^{2}} \frac{2 \Omega_{e}}{k_{y} v_{e}} \mu e^{-\mu} \xi_{e} \sum_{n=-\infty}^{\infty}\left[I_{n}^{\prime}(\mu)-I_{n}(\mu)\right] \xi_{n} Z\left(\xi_{n}\right), \\
D_{y z}= & D_{z y}=k_{y} k_{z} c^{2} / \omega^{2}-\frac{\omega_{p i}^{2}}{\omega^{2}} \xi_{i}\left\{Z\left(\xi_{i}\right) \sin \theta \cos \theta+\left[\xi_{i} \sin \theta+v_{o} / v_{i}\right] Z^{\prime}\left(\xi_{i}\right)\right\} \\
& +\frac{\omega_{p e}^{2}}{\omega^{2}} \frac{2 \Omega_{e}}{k_{y} v_{e}} e^{-\mu} \xi_{e} \sum_{n=-\infty}^{\infty} n I_{n}(\mu) \xi_{n} Z\left(\xi_{n}\right),
\end{aligned}
$$

where $\xi_{i}=\left(\omega-k_{y} v_{o}\right) /\left(k v_{i}\right), \quad \xi_{e}=\omega / k_{z} v_{e}, \quad \xi_{n}=\left(\omega-n \Omega_{e}\right) /\left(k_{z} v_{e}\right), \quad \mu=k_{y}^{2} \rho_{e}^{2} / 2$, $\theta=\cos ^{-1}\left(k_{z} / k\right)$. The other parameters of species $\alpha\left(\alpha=i\right.$ for ions and $e$ for electrons) are $\rho_{\alpha}$ for the gyroradius, $\Omega_{\alpha}$ for gyrofrequency, $\omega_{p \alpha}$ for the plasma frequency, and $v_{\alpha}$ for the thermal speed. The functions $Z$ and $I_{n}$ are the Fried and Conte's plasma dispersion function and the modified Bessel function of order $n$, respectively. Numerical solution of the dispersion equation indicates oblique whistler waves to be excited with a substantial growth rate (a small fraction of the lower hybrid frequency) when the relative motion between ions and electrons constituting the cross-tail current becomes a significant fraction of the ion thermal speed.

Another idea of current disruption is a plasma instability which couples the shear in the plasma flows found in auroral arcs with the co-Iocated plasma pressure gradient (Voronkov et al. 1997). Assuming a velocity shear $V_{y}=V_{o}(x)$, the equation governing this instability is

$$
V_{x}^{\prime \prime}=k^{2} V_{x}\left\{1-\frac{V_{o}^{\prime \prime}}{k\left(\omega-k V_{o}\right)}+\frac{g \rho_{o}^{\prime} / \rho_{o}+g^{2} / V_{f}^{2}}{\left(\omega-k V_{o}\right)^{2}}\right\},
$$


where $g$ is the centripetal acceleration, $\rho_{o}$, is the mass density, and $V_{f}$ is the fast mode velocity. The growth rate is found numerically to be a significant fraction of the velocity shear (KelvinHelmholtz) instability growth rate. The instability onset leads to exponential growth of electric currents along the magnetic field line just like the cross-field current instability. Along a similar line of thought is the excitation of a current-driven instability through the generation of Alfvén waves from strong currents flowing along the magnetic field (Perraut et al. 2000; Le Contel et al. 2001). The growth rate is found to be significant when the relative drift speed associated with the field-aligned current is a small fraction of the electron thermal speed. It is suggested that these Alfvén waves disrupt the field-aligned currents, prompting the cross-tail current system to respond by enhancing the field-aligned currents and causing an apparent diversion of the cross-tail current into the ionosphere.

Another similar idea is based on strong plasma pressure gradient coupled to a positive feedback of electromagnetic waves from the ionosphere, causing a collapse of the magnetic field configuration stretched by the strong cross-tail current (Erickson et al. 2000; Kan et al. 1994; Lyu and Chen 2000). This mechanism is known as the ballooning instability. The theoretical analysis of this has been extended to include particle kinetic effects due to the finite size of ion motion gyrating around the magnetic field and the presence of an electric field along the magnetic field (Cheng and Lui 1998). The critical threshold expressed in terms of plasma beta, which is the ratio between plasma pressure and magnetic field energy density, is

$$
\beta_{c}=\left[1+\frac{b_{i}}{1+b_{i}} \frac{n_{e} T_{e}}{\left(n_{e u t}+n_{e t} H\right) T_{i}}\right] k_{\|}^{2} R_{c} L_{p}+\frac{\omega_{* p i}^{2} R_{c} L_{p}}{4\left(1+b_{i}\right) V_{A}^{2}},
$$

where $b_{i}=\left(k_{\perp} \rho_{i}\right)^{2} / 2, n_{e}$ is the electron number density with subscripts $u$ and $t$ denoting untrapped and trapped populations, $H<<1$ is a dimensionless quantity involving electron distribution weighted by a function of the parallel perturbed electric field, $T_{\alpha}$ is the temperature for species $\alpha, V_{A}$ is the Alfvén speed, $R_{c}$ is the magnetic field curvature, $L_{p}$ is the plasma pressure gradient scale length, and $\omega_{* p i}=v_{i}^{2} \vec{B} \times \nabla P_{i} \bullet k_{\perp} / B \Omega_{i} P_{i}$. The condition in Eq. (9) predicts that the kinetic ballooning instability will be excited when the plasma beta exceeds a value of several tens for parameters pertinent to the magnetotail.

Reduction of electric field in the inner magnetosphere is also considered for the cause of current disruption (Lyons 1996). In the magnetosphere, the earthward motion of particles due to an electric field [see Eq. (3)] competes with the azimuthal motion due to gradient and curvature drifts [see Eqs. (4) and (5)]. Therefore, a reduction in the magnetospheric electric field, presumed to be caused by northward turning of the interplanetary magnetic field, promotes gradient and curvature drifts of magnetospheric particles in the azimuthal direction (westward for ions and eastward for electrons typically). Since higher energy particles drift faster than lower energy ones [Eqs. (4) and (5)], the subsequent buildup of plasma pressure sets up an electric current system resembling the short-circuit of the cross-tail current. Qualitatively, it is shown that 


$$
\frac{\partial j_{11}}{\partial t} \propto \frac{\partial}{\partial t}\left(\frac{\partial P}{\partial y}\right) \propto-\frac{\partial^{2} W}{\partial y^{2}} .
$$

In Eq. (10), $j_{\|}$is the field-aligned current density, $P$ is the plasma pressure, and $W$ is the average plasma energy.

Another proposed mechanism considers the pressure and entropy profiles in the inner magnetosphere (Lee et al. 1998). Sharp plasma pressure develops at the upper and lower edges of the plasma sheet before substorm onset. This in turn leads to a steepening of the entropy profile that enhances the pressure gradient further. The positive feedback generates a plasma instability called the entropy antidiffusion instability, causing relaxation of the magnetic field to a configuration consistent with a less intense cross-tail current.

Each of the above mechanisms can account for some of the observed substorm phenomena and they are usually not mutually exclusive either. In other words, some of these mechanisms can be synthesized to provide a more complete explanation of all the significant substorm features seen. Indeed, attempts for combining these different mechanisms have been made (Lui 1991; Erickson 1995). However, not all these mechanisms are at the same development stage. Some are more conceptual in nature while others are substantiated by detailed theoretical analysis and/or numerical simulation. Much work needs to be done before these plausible mechanisms can be tested and validated. In addition, a major drawback of this current disruption scenario is that global consequences of these different mechanisms are still unconfirmed by global cross-scale simulation, which is a topic at the frontier of research in numerical simulation.

Distinct from the current disruption scenario is the model called the near-Earth neutral line model (Baker et al. 1996). This invokes magnetic reconnection, a process in which magnetic energy in the magnetotail lobes is released by bringing magnetic field lines from opposite magnetotail lobes and joining them together in the plasma sheet via the formation of an Xtype magnetic neutral line (Dungey 1961). The formation of the X-line is envisioned to be due to the tearing instability. The free energy available to drive this instability for a Harris current sheet (with a magnetic field configuration described by $B_{x}=B_{o} \tanh (z / L)$ ) is found to be (Coppi et al. 1966; Schindler 1974)

$$
\delta W=\int d^{3} x\left\{\left|\nabla A_{1}\right|^{2}-\frac{2}{L} \operatorname{sech}^{2}(z / L)\left|A_{1}\right|^{2}+\sum_{\alpha=i, e} 4 \pi T_{\alpha} \int \frac{\left|f_{1 \alpha}\right|^{2}}{f_{o \alpha}} d^{3} v\right\},
$$

where $A_{1}$ is the perturbed vector potential, $f_{o \alpha}$ and $f_{1 \alpha}$ are the initial and perturbed velocity distribution functions for species $\alpha$, respectively. One may visualize the effect of this instability in terms of the cross-tail current breaking up from a sheet form into many individual current filaments aligned along the $x$-axis.

There is much debate on whether or not the tearing instability is stabilized by electron compressibility (Lembége and Pellat 1982; Pellat et al. 1991; Brittnacher et al. 1998; Sitnov et 
al. 1997). This arises from the compression of electrons associated with the formation of current filaments. It may stabilize the tearing instability when the energy required in compressing the electrons exceeds the available free energy from the magnetic field configuration. Nevertheless, if the electron compressibility can be overcome, then plasma sheet particles could be ejected toward the Earth by the magnetic reconnection process caused by the onset of the tearing instability. It is suggested that the slowing down of this earthward plasma transport and the subsequent buildup of the plasma pressure close to the Earth give rise to a current opposing the cross-tail current. Thus, this produces an equivalent effect of a current disruption process (Shiokawa et al. 1997; Birn et al. 1999; Nagai et al. 1998). This scenario is supported by the detection of magnetic reconnection signatures during substorms in the mid-tail region (about 20-30 $R_{E}$ downstream in the magnetotail, $1 R_{E}=6378 \mathrm{~km}$ ) (Birn and Hesse 1996) and by magnetohydrodynamics (MHD) simulations on the consequence of magnetic reconnection in the mid-tail (Nagai et al. 1998). However, observations of current disruption event in the near-Earth magnetotail (within $15 \mathrm{R}_{\mathrm{E}}$ downstream in the magnetotail) do not show preceding earthward plasma flow activity (Lui et al. 1998; Lui et al. 1999) and particle simulation of the braking scenario shows an enhancement of the local current density rather than a reduction as a consequence (Pritchett and Coroniti 2000), contrary to the results from MHD simulations (Nagai et al. 1998). Therefore, further studies from observations, simulation, and theory are needed to substantiate the viability of this scenario.

\section{MAGNETIC STORMS}

A magnetic storm is generally recognized by the time history of the horizontal component of the magnetic field at equatorial latitudes. The official index called Dst (disturbance storm time) is consucted on the basis of this magnetic component from (four) low-latitude magnetic stations. It is now routinely used as a measure of magnetic storm intensity. A magnetic storm generally begins with a sudden increase of the horizontal component of the geomagnetic field (the storm sudden commencement or SSC), followed by a period of the field remaining enhanced, then followed by a period of a substantially reduced field (as much as $\sim 1 \%$ of the Earth's main equatorial field) typically for several hours. The eventual return of the field to its nominal quiet-time value usually takes several days but can last for several weeks. Deviations from the general picture exist, e.g., some storms do not have well defined SSC and the initial phase.

The magnetic field depressions during magnetic storms are caused by the ring current particles encircling the Earth as discussed previously. These charged particles are trapped by the Earth's magnetic field similar to the energetic charged particles in the Van Allen radiation belts (Parks 1991). The charged particle population of the storm-time ring current has been measured directly by spacecraft (Krimigis et al. 1985). The dominant population, with $>95 \%$ of the charged particles in the energy range of $\sim 5-500 \mathrm{keV}$, resides in the radial range of 2 to 8 $R_{E}$ from the Earth.

Another major effect often associated with magnetic storms is the arrival of energetic solar protons. These solar protons can degrade performance of sensors on spacecraft. They can 
also cause radio absorption in the polar region when they bombard the polar ionosphere and increase the ionization at altitudes below the E-layer, leading to abnormally large absorption of radio signals (Bailey 1964). Consequently, a radio blackout in communication can occur in the polar regions. NOAA has established a scale of 1 to 5 , with 5 denoting the most intense storm, for gauging the severity of magnetic storms and their impacts to societal functions. It also provides the service of issuing warnings to interested parties.

At the present time, there are two known drivers for magnetic storms (Lui et al. 2001), as illustrated in Fig. 6. One is enhanced magnetospheric convection from sustained southward interplanetary magnetic field and the other is the accumulated effect of frequent magnetospheric substorms. The mechanism for enhanced convection as the cause is rather simple. Particles residing in the plasma sheet can be transported close to the Earth by a large magnetospheric electric field arising from the interaction of strong southward interplanetary magnetic field with the Earth's magnetic field. The magnetospheric electric field points dawn-to-dusk and the magnetic field is typically northward in the equatorial region of the plasma sheet, resulting in the convection velocity towards the Earth [see Eq. (3)]. Enhancement of magnetospheric electric field allows a deeper penetration of plasma sheet particles earthward by overpowering the azimuthal deflection of particles due to gradient and curvature drifts (Eqs. (4) and (5)).

There are a number of studies yielding results consistent with this view. The Dst index, which is a traditional measure on the strength of magnetic storms, can be predicted well by interplanetary conditions alone (Burton et al. 1975; Kamide et al. 1998). In particular, intense magnetic storms are found during long duration ( $>5 \mathrm{hrs}$ ) of strong southward interplanetary magnetic field, a condition for strong dawn-to-dusk magnetospheric electric field (Gonzalez and Tsurutani 1987). Furthermore, superposed epoch analysis indicates a reduction in the rate of development of the Dst index with substorm occurrence, contrary to the view that substorms contribute to the buildup of the ring current as measured by the Dst index (Iyemori and Rao 1996). Numerical simulations of enhanced magnetospheric convection indeed show buildup of the ring current without the inclusion of impulsive injection from substorms (Chen et al. 1994). It is argued that substorms are incidental to the ring current increase because they are simply another consequence of enhanced magnetospheric convection. However, an important caveat in these numerical simulations is that they invoke a magnetic field model and an electric field model for the calculations of particle trajectory. In other words, these simulations are test particle simulations. The field models employed in the simulations are not obtained in a self-consistent manner with the particle population in the simulation. Therefore, these results need to be confirmed in the future by fully self-consistent numerical simulations.

There is also good evidence to consider storms being caused by frequent occurrence of intense substorms (Akasofu and Chapman 1961). This early view is motivated by observations indicating the presence of frequent substorms during magnetic storms and substantiated further by observations of energetic particles transported impulsively from the plasma sheet into the outer edge of the ring current region during substorms (McIlwain 1974). The subsequent inward displacement of these injected particles can be achieved by radial diffusion. For instance, the radial diffusion coefficient due to electric impulses, e.g., from substorms, is given by (Fälthammar 1965) 


\section{(a) Frequent intense substorms}
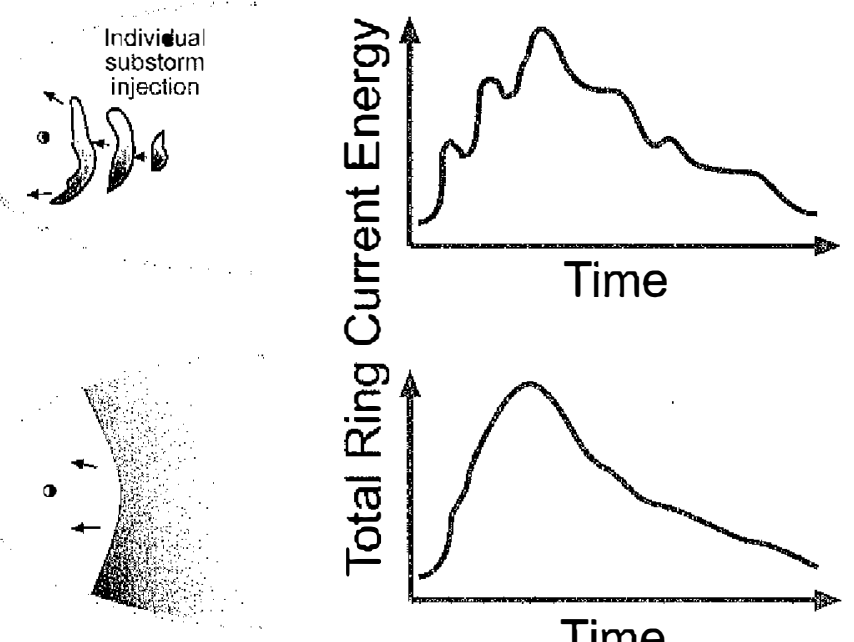

\section{(b) Prolonged strong convection}

Fig. 6. A diagram to illustrate two drivers of magnetic storms.

$$
D_{r}=\frac{r^{6}}{8\left(B_{o} d\right)^{2}} P(\omega)
$$

where $r$ is the geocentric radial distance, $B_{o}$ is the equatorial magnetic field at the subsolar magnetopause at a geocentric distance $d \sim 10 R_{E}$, and $P(\omega)$ is the power spectral density of electric impulses at the frequency $\omega$ of the particle's drift motion around the Earth.

In defense of this second scenario for the cause of magnetic storms, there are counterpoints to the arguments against this view. The argument for substorms being incidental to storm-time ring current buildup can interestingly be turned around to interpret that enhanced magnetospheric convection is another consequence for the condition in which frequent substorms occur. This is because strong southward interplanetary magnetic field is a favorable condition for both strong magnetospheric convection and frequent occurrence of intense substorms. The slower rate of $D s t$ development with substorm occurrence can be explained by the fact that the substorm current system produces the opposite magnetic perturbation as the ring current (Ohtani et al. 2001). Thus, the diminished rate of Dst development simply indicates that the traditional $D s t$ index is not an accurate measure of the ring current strength (Campbell 1996). Furthermore, numerical simulations of the ring current region with impulsive electric pulses mimicking the substorm effect indicate that a stronger ring current than one that can be produced from merely a constant enhanced convection can be generated (Fok et al. 1999). 
A rather convincing case for the importance of substorm in storm development was made with the method of natural orthogonal components (Sun et al. 1998; Sun and Akasofu 2000). This technique uses the magnetic perturbations from a worldwide network of ground magnetometers and decomposes them into spatial and temporal basis functions by solving essentially an eigenfunction equation. Two prominent components were found from this procedure and the associated equivalent ionospheric current system responsible for the ground magnetic perturbations can be constructed. The first component gives a two-cell current pattern well known to be associated with magnetospheric convection. It is in fair correlation with the solar wind parameters but poorly correlated with the $D s t$ index. The second component gives an impulsive one-cell current pattern well known to be associated with substorms. It is less correlated with the solar wind parameters than the first component but is highly correlated with the $D s t$ index. This separation of ground magnetic perturbations into convection and substorm components indicate strongly the important role of substorms in causing storms as indicated by the high correlation with the Dst index.

Recently, a study based on energetic neural atom emission as a measure of the true strength of the ring current shows that both substorms and enhanced magnetospheric convection can contribute to the ring current buildup (Lui et al. 2001). Therefore, a more fruitful direction of storm research than conducting studies to identify a single correct scenario is to evaluate quantitatively the relative importance of these two drivers for magnetic storms in a statistical manner.

\section{CONCLUDING REMARKS}

We conclude this brief review by highlighting some important issues for future investigations. The underlying processes responsible for magnetospheric substorms and magnetic storms are still unresolved. Several plasma instabilities have been proposed for the substorm onset process. Resolving these potential candidates require not only improved theoretical analyses to ascertain the nonlinear consequences of these instabilities for comparison with actual in situ measurements but also global cross-scale simulations to determine the eventual consequences of these instabilities in the entire magnetospheric system. Additionally, better simultaneous measurements in several magnetospheric/ionosphere plasma domains with multiple spacecraft are also essential to determine the coupling between these plasma domains. In terms of magnetic storm research, although two physical mechanisms are presently under consideration, determining the relative importance of these two mechanisms will necessitate a better and direct measure of the storm-time ring current strength and a more quantitative means to evaluate the relative contribution between enhanced magnetospheric convection and substorm in the storm generation.

In addition to these academic issues, significant challenges lie ahead of researchers as magnetospheric physics begins to emerge as an applied science. In order to provide useful and credible service to the society in the space weather area, scientists must identify manageable means to provide accurate nowcasting of space disturbances globally on the Earth's surface as well as within the vast space occupied by the Earth's magnetosphere. On top of that, they must hone their research tools to furnish reliable forecasting needed for present and future space 
endeavors. Large-scale data assimilation and appropriate computer modeling of the magnetosphere will probably form the backbone of these efforts.

Acknowledgements This work was supported by the Atmospheric Sciences Division of the NSF Grant ATM-0000219 and by the NASA Grant NAG5-10475 to the Johns Hopkins University Applied Physics Laboratory.

\section{REFERENCES}

Akasofu, S-I., 1964: The development of the auroral substorm. Planet. Space Sci., 12, 273282.

Akasofu, S. I., and S. Chapman, 1961: The ring current, geomagnetic disturbance and the Van Allen radiation belts. J. Geophys. Res., 66, 1321.

Alfvén, H., 1950: Cosmical Electrodynamics, Oxford University Press.

Bailey, D. K., 1964: Polar cap absorption. Planet. Space Sci., 12, 495.

Baker, D. N., T. I. Pulkkinen, V. Angelopoulos, W. Baumjohann, and R. L. McPherron, 1996: Neutral line model of substorms: Past results and present view. J. Geophys. Res., 101, 12975-13010.

Birn, J., and M. Hesse, 1996: Details of current disruption and diversion in simulations of magnetotail dynamics. J. Geophys. Res., 101, 15345.

Bim, J., M. Hesse, G. Haerendel, W. Baumjohann, and K. Shiokawa, 1999: Flow braking and the substorm current wedge. J. Geophys. Res., 104, 19895-19903.

Brittnacher, M., K. B. Quest, and H. Karimabadi, 1998: A study of the effect of pitch angle and spatial diffusion on tearing instability using a new.finite element based linear code. J. Geophys. Res., 103, 4587-4596.

Burton, R. K., R. L. McPherron, and C. T. Russell, 1975: An empirical relationship between interplanetary conditions and Dst. J. Geophys. Res., 80, 4204-4214.

Campbell, W. H., 1996: Geomagnetic storms, the Dst ring-current myth and lognormal distributions. J. Atm. Terr. Phys., 58, 1171-1181.

Chao, J. K., J. R. Kan, A. T. Y. Lui, and S. I. Akasofu, 1977: A model of thinning of the plasma sheet. Planet. Space Sci., 25, 703-710.

Chen, M. W., M. Schulz, and L. R. Lyons, 1994: Simulations of phase space distributions of stormtime proton ring current. J. Geophys. Res., 99, 5745-5759.

Cheng, C. Z., and A. T. Y. Lui, 1998: Kinetic ballooning instability for substorm onset and current disruption observed by AMPTE/CCE. Geophys. Res. Lett., 25, 4091-4094.

Coppi, B., G. Laval, and R. Pellat, 1966: Dynamics of the geomagnetic tail. Phys. Rev. Lett., 16, 1207-1210.

Dungey, J. W., 1961: Interplanetary magnetic field and the auroral zones. Phys. Res. Lett., 6, 47-48.

Erickson, G. M., 1995: Substorm theories, United they stand, divided they fall. Rev. Geophys., 33, 685-692.

Erickson, G. M., N. C. Maynard, W. J. Burke, G. R. Wilson, and M. A. Heinemann, 2000: 
Electromagnetics of substorm onsets in the near-geosynchronous plasma sheet. $J$. Geophys. Res., 105, 25265-25290.

Fälthammar, C. G., 1965: Effects of time dependent electric fields on geomagnetically trapped radiation. J. Geophys. Res., 70, 2503-2516.

Fok, M. C., T. E. Moore, and D. C. Delcourt, 1999: Modeling of inner plasma sheet and ring current during substorms. J. Geophys. Res., 104, 14557-14569.

Gonzalez, W. D., and B. T. Tsurutani, 1987: Criteria of interplanetary parameters causing intense magnetic storms (Dst <-100 nT). Planet. Space Sci., 35, 1101-1109.

Iyemori, T., and D. R. K. Rao, 1996: Decay of the Dst field of geomagnetic disturbance after substorm onset and its implication to storm-substorm relation. Ann. Geophysicae, 14, 608.

Kamide, Y., W. Baumjohann, I. A. Daglis, W. D. Gonzalez, M. Grande, J. A. Joselyn, R. L. McPherron, J. L. Phillips, G. D. Reeves, G. Rostoker, A. S. Sharma, H. J. Singer, B. T. Tsurutani, and V. M. Vasyliunas, 1998: Current understanding of magnetic storms: Storm-substorm relationships. J. Geophys. Res., 103, 17705-17728.

Kan, J. R., L. H. Lyu, and C. F. Kennel, 1994: Unloading instability triggered by Alfvén waves disrupting the cross-tail current in the near-Earth plasma sheet, in Substorms-2, Proc. of the Second ICS, Fairbanks, Alaska, March 7-11, ed. by J. R. Kan, J. D. Craven, and S. I. Akasofu, 183-188, Geophysical Institute, Univ. of Alaska, Fairbanks.

Krimigis, S. M., G. Gloeckler, R. W. McEntire, T. A. Potemra, F. L. Scarf, and E. G. Shelley, 1985: Magnetic storm of 4 September 1984, A synthesis of ring current spectra and energy densities measured with AMPTE/CCE. Geophys. Res. Lett., 12, 329-332.

Le Contel, O., S. Perraut, A. Roux, and R. Pellat, 2001: Plasma transport during substorm growth phase and relation to breakup. Space Sci. Rev., 95, 415-426.

Lee, L. C., L. Zhang, A. Otto, G. S. Choe, and H. J. Cai, 1998: Entropy antidiffusion instability and formation of a thin current sheet during geomagnetic substorms. J. Geophys. Res., 103, 29419-29428.

Lembége, B., and R. Pellat, 1982: Stability of a thick two-dimensional quasineutral sheet. Phys. Fluids, 25, 1995-2004.

Lui, A. T. Y., 1991: Plasma transport in the Earth's magnetotail. Modeling Magnetospheric Plasma Processes, ed. by G. R. Wilson, 41-53.

Lui, A. T. Y., 1991: A synthesis of magnetospheric substorm models. J. Geophys. Res., 96, $1849-1856$.

Lui, A. T. Y., R. W. McEntire, and K. B. Baker, 2001: A new insight on the cause of magnetic storms. Geophys. Res. Lett., 28, 3413-3416.

Lui, A. T. Y., C. L. Chang, A. Mankofsky, H. K. Wong, and D. Winske, 1991: A cross-field current instability for substorm expansions. J. Geophys. Res., 96, 11389-11401.

Lui, A. T. Y., K. Liou, M. Nosé, S. Ohtani, D. J. Williams, T. Mukai, K. Tsuruda, and S. Kokubun, 1999: Near-Earth dipolarization, Evidence for a non-MHD process. Geophys. Res. Lett., 26, 2905.

Lui, A. T. Y., K. Liou, P. T. Newell, C. I. Meng, S. I. Ohtani, S. Kokubun, T. Ogino, M. Brittnacher, and G. Parks, 1998: Plasma and magnetic flux transport associated with auroral breakups. Geophys. Res. Lett., 25, 4059-4062. 
Lyons, L. R., 1996: Substorms, Fundamental observational features, distinction from other disturbances, and external triggering. J. Geophys. Res., 101, 13011-13026.

Lyu, L. H. and M. Q. Chen, 2000: A kinetic M-I coupling model with unloading instability at onset of substorms. Proc. Fifth International Conference on Substorms, St. Petersburg, Russia, 16-20 May 2000, ESA-SP-443, 315-318.

McIlwain, C. E., 1974: Substorm injection boundaries, in Magnetospheric Physics, ed. by B. M. McCormac, p. 143, D. Reidel, Hingham, Mass.

Nagai, T., M. Fujimoto, Y. Saito, S. Machida, T. Terasawa, R. Nakamura, T. Yamamoto, T. Mukai, A. Nishida, and S. Kokubun, 1998: Structure and dynamics of magnetic reconnection for substorm onsets with Geotail observations. J. Geophys. Res., 103, 4419-4440.

Ohtani, S., M. Nose, G. Rostoker, H. Singer, A. T. Y. Lui, and M. Nakamura, 2001: Stormsubstorm relationship, Contribution of the tail current to Dst. J. Geophys. Res., 106, 21199-21209.

Parks, G. K., 1991: Physics of Space Plasmas, An introduction, Addison-Wesley Publ. Co., Redwood City, CA, U.S.A.

Pellat, R., F. V. Coroniti, and P. L. Pritchett, 1991: Does ion tearing exist? Geophys. Res. Lett., 18, 143-146.

Perraut, S., O. Le Contel, A. Roux, R. Pellat, A. Korth, O. Holter, and A. Pedersen, 2000: Disruption of parallel current at substorm breakup. Geophys. Res. Lett., 27, 4041-4044.

Pritchett, P. L., and F. V. Coroniti, 2000: Localized convection flows and field-aligned current generation in a kinetic model of the near-Earth plasma sheet. Geophys. Res. Lett., 27, 3161-3164.

Schindler, K., 1974: A theory of the substorm mechanism. J. Geophys. Res., 79, 2803-2810.

Shiokawa, K., W. Baumjohann, and G. Haerendel, 1997: Braking of high-speed flows in the near-Earth tail. Geophys. Res. Lett., 24, 1179-1182.

Sitnov, M. I., H. V. Malova, and A. T. Y. Lui, 1997: Quasineutral sheet filamentation instability induced by electron preferential acceleration from stochasticity. J. Geophys. Res., 102, 163-173.

Sun, W., and S. I. Akasofu, 2000: On the forination of the storm-time ring current belt. $J$. Geophys. Res., 105, 5411-5418.

Sun, W., W. Y. Xu, and S. I. Akasofu, 1998: Mathematical separation of directly driven and unloading components in the ionospheric equivalent currents during substorms. $J$. Geophys. Res., 103, 11695-11700.

Voronkov, I., R. Rankin, P. Frycz, V. T. Tikhonchuk, and J. C. Samson, 1997: Coupling of shear flow and pressure gradient instabilities. J. Geophys. Res., 102, 9639-9650. 